Hautarzt 2015 - 66:644-645

DOI 10.1007/s00105-015-3669-y

Online publiziert: 29. August 2015

(c) Springer-Verlag Berlin Heidelberg 2015

\author{
A. Schnuch ${ }^{1} \cdot$ V. Mahler ${ }^{2}$ \\ ${ }^{1}$ IVDK, Universität Göttingen, Göttingen, Deutschland \\ ${ }^{2}$ Allergiebereich, Berufssprechstunde, Gutachten, Hautklinik \\ Universitätsklinikum Erlangen, Erlangen, Deutschland
}

\title{
Volkskrankheit Kontaktallergie
}

Die Kontaktallergie ist eine Volkskrankheit: Epikutantestergebnisse zeigen, dass $15-20 \%$ der Allgemeinbevölkerung gegen mindestens eines der häufigen Kontaktallergene sensibilisiert sind. Manifest an allergischen Kontaktekzemen erkranken mindestens 1-mal in ihrem Leben (Lebenszeitprävalenz) etwa $8 \%$ der erwachsenen Bevölkerung und 5,6\% der Kinder und Jugendlichen. Die relativ hohe Morbidität ist u. a. dadurch bedingt, dass kontaktallergene Stoffe ubiquitär sind: Praktisch alle Bevölkerungsschichten kommen potenziell in Kontakt mit allergenhaltigen Produkten. Eine weitergehende Analyse kann

- unter dem Blickwinkel von Subpopulationen und

- unter dem Blickwinkel der ursächlich bedeutsamen Allergene

erfolgen. In diesem Heft werden die Allergenspektren in den Gruppen der Kinder, der älteren Menschen und der Berufstätigen dargestellt. Durch Einbeziehung des Phänomens der Polysensibilisierung (Sensibilisierung gegen $\geq 3$ Allergene) lässt sich eine Subpopulation mit erhöhtem Risiko für eine Kontaktallergie identifizieren und näher charakterisieren. Die im Epikutantest (ECT) getesteten Allergene selbst verdienen - auch unabhängig epidemiologisch-klinischer Betrachtungsweisen - eine kritische Würdigung ihrer jeweils diagnostischen Treffsicherheit, wobei hier beispielhaft die Diagnostik der Duftstoffallergie behandelt wird.

Betrachtet man die beiden Altersgruppen bei Kindern weiblichen und männlichen Geschlechts getrennt, so zeigen sich in der jungen Altersgruppe keine geschlechtsspezifischen Unterschiede im Sensibilisierungsmuster (außer eines nicht erklärbaren Überwiegens der Wollwachse bei den Jungen). Wenn nun bei den Jugendlichen (13 bis 17 Jahre) weiblichen Geschlechts die Nickel- und Paraphenylendiamin (PPD)-Sensibilisierungen sowohl im Vergleich der jüngeren Altersgruppe als auch im Vergleich zur gleich alten Gruppe der Männer signifikant erhöht war, so lassen sich diese Unterschiede zwanglos mit der zunehmenden Exposition gegenüber Piercing und wohl auch Haarfarben der adoleszenten Frauen erklären. In der Gruppe der 0- bis 12-Jährigen bestand jedoch sehr selten der Verdacht auf eine Allergie durch Haarkosmetika (insbesondere durch Haarfarben) - nämlich in nur $2 \%$ der Getesteten. Und auch in der älteren Gruppe lag dieser Anteil bei nur $10 \%$. Dies macht Alternativerklärungen für die PPD-Sensibilisierungen wahrscheinlich, wonach die wichtigste PPD-Sensibilisierungsquelle im Kindesalter die temporären Henna-Tattoos sind [1]

Bei den über 65-Jährigen zeigt sich ein teilweise abweichendes Sensibilisierungsmuster gegenüber jüngeren Jahrgängen. Uter et al. [2] konnten zeigen, dass dies nicht nur auf altersassoziierte Komorbiditäten wie Ulcus cruris zurückzuführen ist, sondern dass das Alter ein unabhängiger Risikofaktor für vermehrt auftretende Sensibilisierung ist.

\section{) Das Alter ist ein unabhängiger Risikofaktor für vermehrt auftretende Sensibilisierung}

In dem Artikel über Sensibilisierungen bei Senioren wird dem durch Auswertungen der Untergruppen mit und ohne Komorbidität (Ulcus cruris, Unterschenkeldermatitis, chronisch venöse Insuffizienz) Rechnung getragen. Insbesondere die Senioren mit den genannten Komorbiditäten wiesen ein deutlich abweichendes
Sensibilisierungsmuster auf mit auffallend vielen Sensibilisierungen gegen (sehr) schwache Allergene (z. B. Lanolin, Cetearyl Alcohol, Amerchol oder Propylenglykol), die ansonsten kaum eine Rolle spielen. Dies findet in dem ,danger model“ eine plausible Erklärung, wonach vorbestehende Hautentzündungen (z. B. ein Varikosis-bedingtes Unterschenkelekzem) ein Zytokinmilieu bereitstellen, das die Sensibilisierungen fördert [3].

Die Statistik der Duftstoffallergie, die zunächst erkannt wird mit den Epikutantestzubereitungen Duftstoffmix I (DM I), Duftstoffmix II (DM II) und Perubalsam (Myroxylon pereirae), weist die große Gruppe der Duftstoffe wohl als wichtigste Allergene aus. Die Diagnostik der Duftstoffallergie verdient deshalb eine besondere Betrachtung. In dem Artikel von Geier und Uter wird nun erneut gezeigt, dass eine Aufschlüsselung der Mixe im Falle einer positiven Reaktion auf einen Mix unabdingbar ist, um die Diagnostik abzuschließen. Es sind allerdings einige Fallstricke zu beachten: Einfach positive (weniger jedoch stärkere) Reaktionen auf die Mixe gehen allzu oft einher mit negativen Reaktionen auf die Komponenten. Und die Relevanz von „+“-Reaktionen wurde schon früher infrage gestellt: So merkten de Groot und Frosch [4] an: „False-positive reactions (to the Fragrance mix) are not rare, and a single weak (?+ or +) reaction to the mix should not be taken as evidence for fragrance contact allergy but should be substantiated by other tests (e.g. ROAT ...)“ [4].

Andererseits bleiben Patienten, die von vornherein mit den Einzelkomponenten getestet wurden und darauf allergisch reagierten, nicht selten bei der Testung mit dem entsprechenden Mix negativ (falsch negativ; vgl. auch [5]). Mit der Möglichkeit falsch positiver, aber auch falsch ne- 
gativer Reaktionen auf die Mixe bleibt die Diagnostik der Duftstoffallergie eine Herausforderung.

\section{》) Die Kontaktallergie hat eine große sozioökonomische Bedeutung}

Eine besondere Untergruppe der kontaktallergischen Patienten stellen diejenigen mit Verdacht auf Vorliegen einer berufsbedingten Dermatose dar. Gerade hier wird die große sozioökonomische Bedeutung der Kontaktallergie deutlich: Beruflich bedingte Hauterkrankungen führen seit Jahrzehnten die Berufskrankheitenstatistik an. Und wie in dem Artikel von Bauer gezeigt wird, sind es dann bei bestimmten Berufen überwiegend Kontaktallergien, bei anderen eher irritative Ekzeme. Eine Besonderheit der beruflich bedingten Kontaktallergien ist auch das Allergenspektrum, in dem v. a. Gummiinhaltsstoffe (z. B. Thiurame und Mercaptoverbindungen), Biozide (z. B. in Farben) und Epoxidharze (z. B. in Klebern) eine große Rolle spielen. Da eine beruflich bedingte Sensibilisierung durchaus auch die Aufgabe des erlernten Berufes, verbunden mit Umschulungen oder Berentungen, nach sich ziehen kann, ist eine noxenbezogene Primärprävention, die die Ausschaltung oder mengenmäßige Verminderung der Allergenexposition zum Ziel hat, von größter Bedeutung [6]. Dies setzt allerdings ein kontinuierliches Allergenmonitoring voraus.

Während bisher die Allergene als Hauptverursacher der Kontaktallergie im Fokus standen, wendet sich der Artikel von Schwitulla et al. einer Gruppe von Patienten zu, die sich durch eine besondere Suszeptibilität auszeichnet. Es handelt sich dabei um die Polysensibilisierten, und bisherige Untersuchungen sprechen dafür, dass die gesteigerte Suszeptibilität eine genetische Grundlage haben könnte [7]. Schwitulla et al. untersuchten nun klinische und demografische Faktoren, die mit einer Polysensibilisierung assoziiert sein können. Identifiziert wurden Alter, Geschlecht und Ekzemlokalisation, aber auch bestimmte Sensibilisierungen (vor allem schwächere Sensibilisatoren wie Cetearyl Alcohol oder Parabene [8]).
Zum Abschluss sei angemerkt, dass alle hier präsentierten Artikel auf den Datenbestand des multizentrischen Projektes Informationsverbund Dermatologischer Kliniken (IVDK; www.ivdk.org) zurückgreifen konnten. Im IVDK kooperieren seit über 25 Jahren Allergieabteilungen aus Deutschland, Österreich und der Schweiz, zurzeit 56, die gemeinsam nach den Standards und Empfehlungen der Deutschen Kontaktallergie-Gruppe arbeiten [9]. Während der IVDK seine Hauptaufgabe in der Überwachung der Kontaktallergie sieht, ermöglicht seine Registerfunktion mit Daten von $>240.000 \mathrm{~Pa}$ tienten retrospektive Analysen, wie hier beispielhaft gezeigt [10].

Insgesamt ergibt sich also bei näherer Betrachtung des Phänomens Kontaktallergie ein heterogenes, facettenreiches Bild [11], dem man z. B. mit der bloßen Beschränkung auf die „Hitliste“ der Allergene nicht gerecht wird, gemäß der Erfahrung, dass zu grobe Simplifizierungen komplexer Zusammenhänge zum Verlust von Information führt. Dies sollte hier vermieden werden.

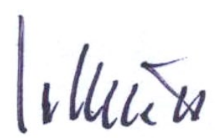

Prof. Dr. Axel Schnuch Leiter des IVDK

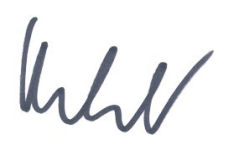

Prof. Dr. Vera Mahler

Vorsitzende der DKG

\section{Korrespondenzadresse}

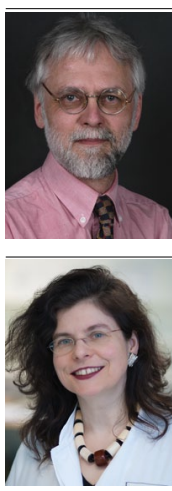

Prof. Dr. A. Schnuch

IVDK

Universität Göttingen

von Siebold Str. 3

37075 Göttingen

aschnuc@gwdg.de

\section{Prof. Dr. V. Mahler}

Allergiebereich,

Berufssprechstunde,

Gutachten,

Hautklinik Universitätsklinikum

Erlangen, Ulmenweg 18

91054 Erlangen

Vera.mahler@uk-erlangen.de
Interessenkonflikt. Der IVDK wird von der Kosmetikindustrie finanziell unterstützt. A. Schnuch ist als Ad-hoc-Berater der Kosmetikindustrie tätig, z. T. honoriert. V. Mahler ist Vorsitzende der Deutschen Kontaktallergie-Gruppe, einer wissenschaftlichen Non-Profit-Organisation, die in den letzten 5 Jahren Spenden der Firmen Almirall-Hermal und SmartPractice Germany erhalten hat. V. Mahler hat Referentenhonorare von Fa. Almirall-Hermal und SmartPractice erhalten.

\section{Literatur}

1. Spornraft-Ragaller P, Schnuch A, Uter W (2011) Extreme patch test reactivity to $\mathrm{p}$-phenylenediamine but not to other allergens in children. Contact Dermatitis 65:220-226

2. Uter W, Geier J, Pfahlberg A, Effendy I (2002) The spectrum of contact allergy in elderly patients with and without lower leg dermatitis. Dermatology 204:266-272

3. McFadden JP, Basketter DA (2000) Contact allergy, irritancy and, danger.' Contact Dermatitis 42:123127

4. DeGroot AC, Frosch PH (1997) Adverse reactions to fragrances. A clinical review. Contact Dermatitis 36:57-86

5. Mann J, McFadden JP, White JML, White IR, Banerjee $P$ (2014) Baseline series fragrance markers fail to predict contact allergy. Contact Dermatitis 70:276-281

6. Uter W, Geier J, Schnuch A (2008) Der Erfolg noxenbezogener Primärprävention. Nachweis am Beispiel der Kontaktallergie-Überwachung. Gefahrstoffe - Reinhalt Luft 68:202-208

7. Schnuch A, Westphal G, Mössner R, Uter W, Reich $K$ (2011) Genetic factors in contact allergy - review and future goals. Contact Dermatitis 64:2-23

8. Schnuch A, Brasch J, Lessmann H, Geier J, Uter W (2007) A further characteristic of susceptibility to contact allergy: sensitization to a weak contact allergen is associated with polysensitization. Results of the IVDK. Contact Dermatitis 56:331-337

9. Mahler V, Geier J, Schnuch A (2014) Neue Entwicklungen zum Thema Epikutantest - aktuelle Daten aus DKG und IVDK. J Dtsch Dermatol Ges 12:583592

10. Schnuch A, Geier J, Lessmann H, Arnold R, Uter W (2012) Surveillance of contact allergies: methods and results of the Information Network of Departments of Dermatology (IVDK). Allergy 67:847-857

11. Mahler V (2014) Kontaktekzeme. Akt Dermatol 40:95-107 